The NMR spectral data for compounds 3 and 10 were incorrect in the version published March 10, 2005; the corrected version was published March 6, 2006.

\title{
Synthesis of a Potent and Selective Inhibitor of p90 Rsk
}

David J. Maloney, and Sidney M. Hecht* 
General Methods. Reagents and solvents were reagent grade and used without further purification.

Methylene chloride was distilled from calcium hydride, and toluene was distilled from sodium. Anhydrous grade THF and $\mathrm{Et}_{2} \mathrm{O}$ were purchased from VWR. All reactions involving air or moisture sensitive reagents or intermediates were performed under a nitrogen or argon atmosphere. Flash chromatography was performed using Silicycle 40-60 mesh silica gel. Analytical TLC was performed using 0.25 mm EM silica gel $60 \mathrm{~F}_{250}$ plates that were visualized by irradiation $(254 \mathrm{~nm})$ or by staining with Hanessian's stain (cerium molybdate). Optical rotations were obtained using a Jasco digital polarimeter. ${ }^{1} \mathrm{H}$ and ${ }^{13} \mathrm{C}$ NMR spectra were obtained using $300 \mathrm{MHz}$ and $500 \mathrm{MHz}$ Varian instruments. Chemical shifts are reported in parts per million $\left(\mathrm{ppm} \delta\right.$ ) referenced to the residual ${ }^{1} \mathrm{H}$ resonance of the solvent $\left(\mathrm{CDCl}_{3}, 7.26 \mathrm{ppm}\right.$; DMSO- $d_{6}, 2.49$

ppm). ${ }^{13} \mathrm{C}$ spectra were referenced to the residual ${ }^{13} \mathrm{C}$ resonance of the solvent $\left(\mathrm{CDCl}_{3}, 77.3 \mathrm{ppm}\right.$; DMSO- $d_{6}$, $39.5 \mathrm{ppm}$ ). Splitting patterns are designated as follows: s, singlet; br, broad; d, doublet; dd, doublet of doublets; t, triplet; q, quartet; m, mulitplet. High resolution mass spectra were obtained at the Michigan State University-NIH Mass Spectrometry Facility. Melting points were determined for all crystalline compounds and are uncorrected.

\section{Experimental Procedures.}

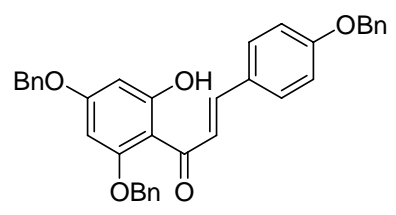

E-3-(4-Benzyloxyphenyl)-1-(2,4-bisbenzyloxy-6-hydroxyphenyl)propenone (2). To a solution containing $5.00 \mathrm{~g}$ (18.4 mmol) of 4',5,7-trihydroxyflavanone in $150 \mathrm{~mL}$ of acetone was added $10.0 \mathrm{~g}(7.00 \mathrm{~mL}, 57.0$ mmol) of $\mathrm{BnBr}$ followed by $15.4 \mathrm{~g}(110 \mathrm{mmol})$ of $\mathrm{K}_{2} \mathrm{CO}_{3}$. The reaction mixture was heated at reflux overnight under $\mathrm{N}_{2}$. The cooled reaction mixture was then concentrated under diminished pressure. The residue was redissolved in $300 \mathrm{~mL}$ of ethyl acetate, washed with three $100-\mathrm{mL}$ portions of $\mathrm{H}_{2} \mathrm{O}$, dried $\left(\mathrm{MgSO}_{4}\right)$ and concentrated under diminished pressure. The residue was purified by flash chromatography on 
a silica gel column $(28 \times 4 \mathrm{~cm})$. Elution with 3:1 hexanes-ethyl acetate gave 2 as a bright yellow solid: yield $8.07 \mathrm{~g}(81 \%) ; \mathrm{mp} 127-129{ }^{\circ} \mathrm{C}$; silica gel TLC $R_{f} 0.53$ (3:1 hexanes-ethyl acetate); ${ }^{1} \mathrm{H}$ NMR $\left(\mathrm{CDCl}_{3}\right) \delta 5.02$ $(\mathrm{s}, 2 \mathrm{H}), 5.07(\mathrm{~s}, 4 \mathrm{H}), 6.15(\mathrm{~d}, 1 \mathrm{H}, J=2.1 \mathrm{~Hz}), 6.21(\mathrm{~d}, 1 \mathrm{H}, J=2.1 \mathrm{~Hz}), 6.76(\mathrm{~d}, 2 \mathrm{H}, J=8.4 \mathrm{~Hz}), 6.97(\mathrm{~d}, 2 \mathrm{H}$, $J=8.7 \mathrm{~Hz}), 7.32(\mathrm{~m}, 15 \mathrm{H}), 7.74(\mathrm{dd}, 2 \mathrm{H}, J=15.3,14.7 \mathrm{~Hz})$ and $14.81(\mathrm{br} \mathrm{s}, 1 \mathrm{H}) ;{ }^{13} \mathrm{C} \mathrm{NMR}\left(\mathrm{CDCl}_{3}\right) \delta 70.3$, $70.5,71.6,92.7,95.3,106.5,115.2,125.4,127.5,127.7,127.9,128.3,128.4,128.6,128.8,128.9,129.1$, $129.2,130.4,135.7,136.1,136.8,143.0,160.5,161.9,165.3,169.1$ and 192.8; mass spectrum $(\mathrm{FAB}), \mathrm{m} / \mathrm{z}$ 543.2177 $(\mathrm{M}+\mathrm{H})^{+}\left(\mathrm{C}_{36} \mathrm{H}_{31} \mathrm{O}_{5}\right.$ requires 543.2171).

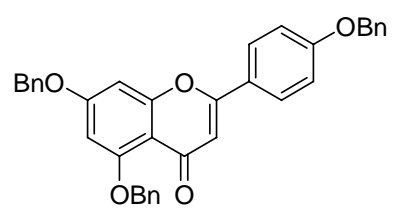

5,7-Bis-(benzyloxy)-2-(4-(benzyloxy)phenyl)-4H-chromen-4-one (3). To a solution containing $7.50 \mathrm{~g}$ (13.8 mmol) of 2 in $50 \mathrm{~mL}$ of DMSO was added $0.39 \mathrm{~g}(1.52 \mathrm{mmol})$ of $\mathrm{I}_{2}$. The reaction mixture was heated at reflux overnight under $\mathrm{N}_{2}$. The cooled reaction mixture was diluted with $200 \mathrm{~mL}$ of ethyl acetate and washed with two $100-\mathrm{mL}$ portions of $1 \mathrm{~N} \mathrm{HCl}$. The organic layer was separated, dried $\left(\mathrm{MgSO}_{4}\right)$ and concentrated under diminished pressure. The residue was purified by flash chromatography on a silica gel column $(28 \times 4 \mathrm{~cm})$. Elution with 1:1 hexanes-ethyl acetate gave 3 as an off-white solid: yield 5.50 g (72\%); mp 60-62 ${ }^{\circ} \mathrm{C}$; silica gel TLC $R_{f} 0.19\left(2: 1\right.$ hexanes-ethyl acetate); ${ }^{1} \mathrm{H}$ NMR $\left(\mathrm{CDCl}_{3}\right) \delta 5.10(\mathrm{~s}, 2 \mathrm{H}), 5.12(\mathrm{~s}$, 2H), $5.22(\mathrm{~s}, 2 \mathrm{H}), 6.48(\mathrm{~d}, 1 \mathrm{H}, J=2.5 \mathrm{~Hz}), 6.57(\mathrm{~s}, 1 \mathrm{H}), 6.60(\mathrm{~d}, 1 \mathrm{H}, J=2.5 \mathrm{~Hz}), 7.05(\mathrm{~d}, 2 \mathrm{H}, J=8.5 \mathrm{~Hz})$, 7.37-7.46 (m, 13H), 7.64 (d, 2H, $J=8.0 \mathrm{~Hz})$ and $7.80(\mathrm{~d}, 2 \mathrm{H}, J=9.0 \mathrm{~Hz}) ;{ }^{13} \mathrm{C} \mathrm{NMR}\left(\mathrm{CDCl}_{3}\right) \delta 70.2,70.6$, $70.8,94.3,98.4,107.8,109.9,115.3,124.1,126.7,127.6,127.76,128.36,128.55,128.71,128.84,128.89$, $139.0,136.4,136.6,159.7,159.8,160.7,161.3,163.0$ and 177.4; mass spectrum $(\mathrm{FAB}), \mathrm{m} / \mathrm{z} 541.2019(\mathrm{M}+$ $\mathrm{H})^{+}\left(\mathrm{C}_{36} \mathrm{H}_{29} \mathrm{O}_{5}\right.$ requires 541.2015).

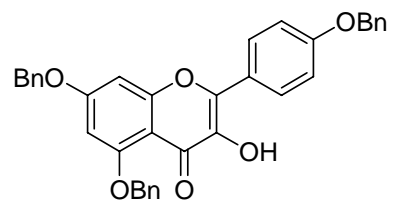


5,7-Bis-(benzyloxy)-2-(4-(benzyloxy)phenyl)-3-hydroxy-4H-chromen-4-one (4). To a solution containing $1.00 \mathrm{~g}(1.85 \mathrm{mmol})$ of $3 \mathrm{in} 30 \mathrm{~mL}$ of $\mathrm{CH}_{2} \mathrm{Cl}_{2}$ at $0{ }^{\circ} \mathrm{C}$ was added $30 \mathrm{~mL}$ of a $0.9-0.11 \mathrm{M}$ solution of DMDO in acetone. The reaction mixture was stirred at $0{ }^{\circ} \mathrm{C}$ overnight under $\mathrm{N}_{2}$. The solvent was concentrated under diminished pressure, then the residue was redissolved in $50 \mathrm{~mL}$ of $\mathrm{CH}_{2} \mathrm{Cl}_{2}$ and treated with catalytic pTsOH. The reaction mixture was stirred at $0{ }^{\circ} \mathrm{C}$ for $30 \mathrm{~min}$. The solvent was concentrated under diminished pressure and the residue was purified by flash chromatography on a silica gel column $(25 \mathrm{x} 4 \mathrm{~cm})$. Elution with 1:1 hexanes-ethyl acetate gave 4 as a light brown solid: yield $0.63 \mathrm{~g}$ ( $78 \%$ based on consumed starting material) and $0.22 \mathrm{~g}$ of unreacted starting material; silica gel TLC $R_{f} 0.28$ (3:1 hexanes-ethyl acetate); ${ }^{1} \mathrm{H}$ NMR (DMSO-d $) \delta 5.19(\mathrm{~s}, 2 \mathrm{H}), 5.25(\mathrm{~s}, 4 \mathrm{H}), 6.69(\mathrm{~s}, 1 \mathrm{H}), 6.97(\mathrm{br} \mathrm{s}, 1 \mathrm{H}), 7.18(\mathrm{~d}, 2 \mathrm{H}, J=8.5 \mathrm{~Hz}), 7.30-$ $7.44(\mathrm{~m}, 11 \mathrm{H}), 7.46-7.50(\mathrm{~m}, 2 \mathrm{H}), 7.67(\mathrm{~d}, 2 \mathrm{H}, J=7.5 \mathrm{~Hz}), 8.14(\mathrm{~d}, 2 \mathrm{H}, J=8.5 \mathrm{~Hz})$ and $9.15(\mathrm{~s}, 1 \mathrm{H}) ;{ }^{13} \mathrm{C}$ NMR $\left(\mathrm{DMSO}-d_{6}\right) \delta 70.1,70.5,70.7,94.6,98.0,102.2,107.4,115.6,127.2,128.1,128.5,128.6,128.7$, $128.87,128.92,129.16,129.23,129.4,136.8,137.4,137.6,138.8,142.5,158.7,159.5,159.8,163.4$ and 171.9; mass spectrum $(\mathrm{FAB}), \mathrm{m} / \mathrm{z} 557.1967(\mathrm{M}+\mathrm{H})^{+}\left(\mathrm{C}_{36} \mathrm{H}_{29} \mathrm{O}_{6}\right.$ requires 557.1964).

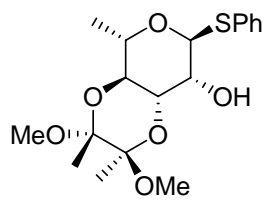

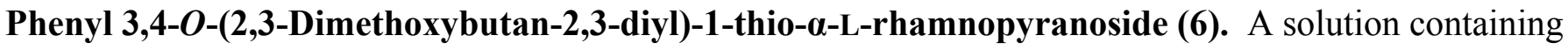
$3.80 \mathrm{~g}(14.8 \mathrm{mmol})$ of $5,1.40 \mathrm{~g}(1.42 \mathrm{~mL}, 16.3 \mathrm{mmol})$ of 2,3-butanedione, $4.72 \mathrm{~g}(4.87 \mathrm{~mL}, 44.5 \mathrm{mmol})$ of trimethylorthoformate, and $0.52 \mathrm{~g}$ (2.22 mmol) 10-camphorsulfonic acid in $100 \mathrm{~mL}$ of $\mathrm{MeOH}$ was heated at reflux overnight. The cooled reaction mixture was neutralized with $\mathrm{NEt}_{3}$ and concentrated under diminished pressure. The residue was purified by flash chromatography on a silica gel column $(28 \mathrm{x} 4 \mathrm{~cm})$. Elution with 6:1 hexanes-ethyl acetate gave 6 as a colorless foam: yield $4.87 \mathrm{~g}(89 \%)$; silica gel TLC $R_{f} 0.19(3: 1$ hexanes-ethyl acetate); $[\alpha]_{\mathrm{D}}{ }^{23}-290.9\left(\mathrm{c} 1.36, \mathrm{CHCl}_{3}\right) ;{ }^{1} \mathrm{H} \mathrm{NMR}\left(\mathrm{CDCl}_{3}\right) \delta 1.26(\mathrm{~d}, 3 \mathrm{H}, J=6.3 \mathrm{~Hz}), 1.31(\mathrm{~s}$, $3 \mathrm{H}), 1.33(\mathrm{~s}, 3 \mathrm{H}), 2.83(\mathrm{br} \mathrm{s}, 1 \mathrm{H}), 3.24(\mathrm{~s}, 3 \mathrm{H}), 3.31(\mathrm{~s}, 3 \mathrm{H}), 3.78(\mathrm{~m}, 1 \mathrm{H}), 3.98(\mathrm{dd}, 1 \mathrm{H}, J=10.2,3.0 \mathrm{~Hz})$, $4.18(\mathrm{dd}, 1 \mathrm{H}, J=3.0,1.2 \mathrm{~Hz}), 4.26(\mathrm{~m}, 1 \mathrm{H}), 5.49(\mathrm{~s}, 1 \mathrm{H}), 7.26-7.32(\mathrm{~m}, 3 \mathrm{H})$ and $7.42-7.47(\mathrm{~m}, 2 \mathrm{H}) ;{ }^{13} \mathrm{C}$ 
$\operatorname{NMR}\left(\mathrm{CDCl}_{3}\right) \delta 16.7,17.9,18.0,47.9,48.3,68.0,68.8,69.0,71.6,88.1,100.1,100.5,127.5,129.2,131.6$ and 134.5; mass spectrum (FAB), $\mathrm{m} / \mathrm{z} 370.1448(\mathrm{M})^{+}\left(\mathrm{C}_{18} \mathrm{H}_{26} \mathrm{O}_{6} \mathrm{~S}\right.$ requires 370.1450$)$.

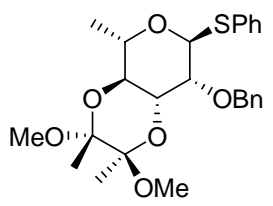

Phenyl 2-O-Benzyl-3,4-O-(2,3-dimethoxybutan-2,3-diyl)-1-thio- $\alpha$-L-rhamnopyranoside. To a solution containing $4.80 \mathrm{~g}(13.0 \mathrm{mmol})$ of 6 in $100 \mathrm{~mL}$ of anhydrous THF at $0{ }^{\circ} \mathrm{C}$ was added slowly $1.03 \mathrm{~g}(25.9$ $\mathrm{mmol}$ ) of a $60 \%$ dispersion of $\mathrm{NaH}$. After stirring for $5 \mathrm{~min}$, the reaction mixture was removed from the ice bath and $3.33 \mathrm{~g}(2.31 \mathrm{~mL}, 19.5 \mathrm{mmol})$ of $\mathrm{BnBr}$ was added. The reaction mixture was heated at reflux overnight under $\mathrm{N}_{2}$. The reaction mixture was then quenched by the slow dropwise addition of sat. aqueous $\mathrm{NH}_{4} \mathrm{Cl}$, diluted with $200 \mathrm{~mL}$ of $\mathrm{H}_{2} \mathrm{O}$, and extracted with three $150-\mathrm{mL}$ portions of ethyl acetate. The combined organic layer was dried $\left(\mathrm{MgSO}_{4}\right)$ and concentrated under diminished pressure. The residue was purified by flash chromatography on a silica gel column $(28 \times 4 \mathrm{~cm})$. Elution with 4:1 hexanes-ethyl acetate gave the product as a colorless foam: yield $5.05 \mathrm{~g}(85 \%)$; silica gel TLC $R_{f} 0.62$ (3:1 hexanes-ethyl acetate); $[\alpha]_{\mathrm{D}}{ }^{21}-206.7\left(\mathrm{c} 1.08, \mathrm{CHCl}_{3}\right) ;{ }^{1} \mathrm{H} \mathrm{NMR}\left(\mathrm{CDCl}_{3}\right) \delta 1.29(\mathrm{~d}, 3 \mathrm{H}, J=6.3 \mathrm{~Hz}), 1.34(\mathrm{~s}, 3 \mathrm{H}), 1.36(\mathrm{~s}, 3 \mathrm{H}), 3.29$ $(\mathrm{s}, 3 \mathrm{H}), 3.31(\mathrm{~s}, 3 \mathrm{H}), 3.95(\mathrm{~m}, 3 \mathrm{H}), 4.26(\mathrm{~m}, 1 \mathrm{H}), 4.71(\mathrm{~m}, 1 \mathrm{H}), 4.90(\mathrm{~d}, 1 \mathrm{H}, J=12.0 \mathrm{~Hz}), 5.45(\mathrm{~d}, 1 \mathrm{H}, J=$ $0.6 \mathrm{~Hz})$ and 7.25-7.45 (m, 10H); ${ }^{13} \mathrm{C} \mathrm{NMR}\left(\mathrm{CDCl}_{3}\right) \delta 16.8,18.0,18.1,47.9,48.1,68.4,69.1,69.6,73.2$, $77.9,87.5,99.8,100.0,127.3,127.7,128.1,128.4,129.2,131.3,135.0$ and 138.6; mass spectrum $(\mathrm{FAB}), \mathrm{m} / \mathrm{z}$ $460.1919(\mathrm{M})^{+}\left(\mathrm{C}_{25} \mathrm{H}_{32} \mathrm{O}_{6} \mathrm{~S}\right.$ requires 460.1920).

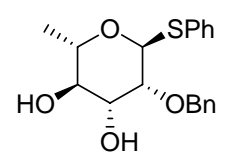

Phenyl 2-O-Benzyl-1-thio-a-L-rhamnopyranoside (7). To a solution containing $5.00 \mathrm{~g}$ (10.87 mmol) of phenyl 2-O-benzyl-3,4-O-(2,3-dimethoxybutan-2,3-diyl)-1-thio- $\alpha$-L-rhanmopyranoside in $100 \mathrm{~mL}$ of $\mathrm{CH}_{2} \mathrm{Cl}_{2}$ was added $22 \mathrm{~mL}$ of 10:1 TFA $-\mathrm{H}_{2} \mathrm{O}$. The reaction mixture was stirred at room temperature for $40 \mathrm{~min}$, quenched cautiously with sat. aq $\mathrm{NaHCO}_{3}$, and diluted with $75 \mathrm{~mL}$ of $\mathrm{CH}_{2} \mathrm{Cl}_{2}$. The organic layer was 
separated, washed with two 100-mL portions of brine, dried $\left(\mathrm{MgSO}_{4}\right)$ and concentrated under diminished pressure. The residue was purified by flash chromatography on a silica gel column $(28 \mathrm{x} 4 \mathrm{~cm})$. Elution with 1:1 hexanes-ethyl acetate gave 7 as a colorless solid: yield $3.50 \mathrm{~g}(93 \%)$; silica gel TLC $R_{f} 0.47(1: 1$

hexanes-ethyl acetate); $[\alpha]_{\mathrm{D}}{ }^{21}-92.1\left(\right.$ c $\left.1.00, \mathrm{CHCl}_{3}\right) ;{ }^{1} \mathrm{H} \mathrm{NMR}\left(\mathrm{CDCl}_{3}\right) \delta 1.34(\mathrm{~d}, 3 \mathrm{H}, J=6.0 \mathrm{~Hz}), 2.42(\mathrm{br}$ s, 2H), $3.52(\mathrm{t}, 1 \mathrm{H}, J=9.5 \mathrm{~Hz}), 3.76(\mathrm{dd}, 1 \mathrm{H}, J=9.0,3.5 \mathrm{~Hz}), 4.01(\mathrm{dd}, 1 \mathrm{H}, J=3.5,1.5 \mathrm{~Hz}), 4.13(\mathrm{~m}, 1 \mathrm{H})$, $4.49(\mathrm{~d}, 1 \mathrm{H}, J=11.5 \mathrm{~Hz}), 4.75(\mathrm{~d}, 1 \mathrm{H}, J=12.0 \mathrm{~Hz}), 5.60(\mathrm{~s}, 1 \mathrm{H}), 7.29(\mathrm{~m}, 8 \mathrm{H})$ and $7.47(\mathrm{~m}, 2 \mathrm{H}) ;{ }^{13} \mathrm{C} \mathrm{NMR}$ $\left(\mathrm{CDCl}_{3}\right) \delta 17.9,69.3,72.3,72.6,74.5,79.8,85.2,127.7,128.2,128.4,128.9,129.3,131.8,134.4$ and 137.3 mass spectrum $(\mathrm{FAB}), \mathrm{m} / \mathrm{z} 346.1237(\mathrm{M})^{+}\left(\mathrm{C}_{19} \mathrm{H}_{22} \mathrm{O}_{4} \mathrm{~S}\right.$ requires 346.1239).

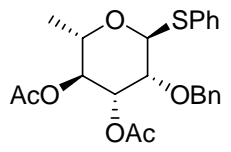

Phenyl 3,4-Di-O-acetyl-2-O-benzyl-1-thio-o-L-rhamnopyranoside (8). A solution containing 3.45 g (9.97 $\mathrm{mmol})$ of 7, $4.07 \mathrm{~g}(3.77 \mathrm{~mL}, 39.9 \mathrm{mmol})$ of $\mathrm{Ac}_{2} \mathrm{O}, 5.03 \mathrm{~g}(6.78 \mathrm{~mL}, 49.9 \mathrm{mmol})$ of $\mathrm{NEt}_{3}$, and $0.13 \mathrm{~g}(0.10$ mmol) of 4-(N,N-dimethyl)aminopyridine in $75 \mathrm{~mL}$ of anhyd $\mathrm{CH}_{2} \mathrm{Cl}_{2}$ was stirred at room temperature overnight under $\mathrm{N}_{2}$. The reaction mixture was then diluted with $100 \mathrm{~mL}$ of $\mathrm{CH}_{2} \mathrm{Cl}_{2}$ and washed with two 100-mL portions of $\mathrm{H}_{2} \mathrm{O}$. The organic layer was separated, dried $\left(\mathrm{MgSO}_{4}\right)$ and concentrated under diminished pressure. The residue was purified by flash chromatography on a silica gel $(25 \mathrm{x} 4 \mathrm{~cm})$. Elution with 2:1 hexanes-ethyl acetate gave 8 as a colorless solid: yield $4.30 \mathrm{~g}(100 \%)$; silica gel TLC $R_{f} 0.51(2: 1$ hexanes-ethyl acetate); $[\alpha]_{\mathrm{D}}{ }^{21}-43.3\left(\mathrm{c} 1.56, \mathrm{CHCl}_{3}\right) ;{ }^{1} \mathrm{H} \mathrm{NMR}\left(\mathrm{CDCl}_{3}\right) \delta 1.24(\mathrm{~d}, 3 \mathrm{H}, \mathrm{J}=6.0 \mathrm{~Hz}), 1.99(\mathrm{~s}$, 3H), $2.07(\mathrm{~s}, 3 \mathrm{H}), 4.08(\mathrm{dd}, 1 \mathrm{H}, J=3.5,1.5 \mathrm{~Hz}), 4.28(\mathrm{~m}, 1 \mathrm{H}), 4.54(\mathrm{~d}, 1 \mathrm{H}, J=12.5 \mathrm{~Hz}), 4.68(\mathrm{~d}, 1 \mathrm{H}, J=$ $12.0 \mathrm{~Hz}), 5.16(\mathrm{dd}, 1 \mathrm{H}, J=10.0,3.5 \mathrm{~Hz}), 5.27(\mathrm{t}, 1 \mathrm{H}, J=9.5 \mathrm{~Hz}), 5.50(\mathrm{~d}, 1 \mathrm{H}, J=2.0 \mathrm{~Hz}), 7.27-7.33(\mathrm{~m}$, $8 \mathrm{H})$ and $7.44(\mathrm{~m}, 2 \mathrm{H}) ;{ }^{13} \mathrm{C} \mathrm{NMR}\left(\mathrm{CDCl}_{3}\right) \delta 17.7,21.1,68.0,71.6,71.7,72.8,77.2,85.6,127.7,128.2,128.2$, 128.7, 129.4, 131.6, 134.3, 137.6, 170.2 and 170.5; mass spectrum (FAB), $\mathrm{m} / \mathrm{z}$ 431.1530 $(\mathrm{M}+\mathrm{H})^{+}$ $\left(\mathrm{C}_{23} \mathrm{H}_{27} \mathrm{O}_{6} \mathrm{~S}\right.$ requires 431.1528$)$.

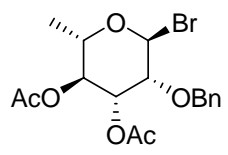


3,4-Di-O-acetyl-2-O-benzyl-a-L-rhamnopyranosyl Bromide (9). To a solution containing $1.00 \mathrm{~g}$ (2.32 mmol) of 8 in $25 \mathrm{~mL}$ of anhydrous $\mathrm{CH}_{2} \mathrm{Cl}_{2}$ at $0{ }^{\circ} \mathrm{C}$ under argon was added $0.50 \mathrm{~g}(0.16 \mathrm{~mL}, 3.14 \mathrm{mmol})$ of $\mathrm{Br}_{2}$. The reaction mixture was stirred at $0{ }^{\circ} \mathrm{C}$ for $1 \mathrm{~h}$. The reaction mixture was diluted with $20 \mathrm{~mL}$ of $\mathrm{CH}_{2} \mathrm{Cl}_{2}$ and washed with $100 \mathrm{~mL}$ of $3 \%$ aq $\mathrm{NaHSO}_{3}$. The organic layer was separated, dried $\left(\mathrm{MgSO}_{4}\right)$ and concentrated under diminished pressure. The residue was then purified by flash chromatography on a silica gel column $(26 \times 4 \mathrm{~cm})$. Elution with 2:1 hexanes-ethyl acetate gave 9 as a colorless oil: yield $0.78 \mathrm{~g}(84 \%)$; silica gel TLC $R_{f} 0.21\left(2: 1\right.$ hexanes-ethyl acetate); ${ }^{1} \mathrm{H}$ NMR $\left(\mathrm{CDCl}_{3}\right) \delta 1.26(\mathrm{~d}, 3 \mathrm{H}, J=6.0 \mathrm{~Hz}), 1.99(\mathrm{~s}, 3 \mathrm{H})$, $2.07(\mathrm{~s}, 3 \mathrm{H}), 4.03(\mathrm{~m}, 1 \mathrm{H}), 4.10(\mathrm{~m}, 1 \mathrm{H}), 4.66(\mathrm{dd}, 2 \mathrm{H}, J=15.0,12.0 \mathrm{~Hz}), 5.23(\mathrm{t}, 1 \mathrm{H}, J=10.2 \mathrm{~Hz}), 5.54$ $(\mathrm{dd}, 1 \mathrm{H}, J=10.2,3.3 \mathrm{~Hz}), 6.34(\mathrm{~s}, 1 \mathrm{H})$ and $7.35(\mathrm{~m}, 5 \mathrm{H}) ;{ }^{13} \mathrm{C} \mathrm{NMR}\left(\mathrm{CDCl}_{3}\right) \delta 17.3,21.0,70.0,70.8,71.4$, 73.5, 79.2, 86.5, 128.2, 128.8, 137.4, 169.9 and 170.2. Note: must be used immediately; product decomposes rather rapidly (1-2 days).

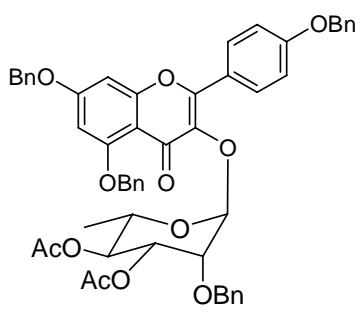

\section{5,7-Bis-(benzyloxy)-2-(4-(benzyloxy)phenyl)-3-[3,4-di-O-acetyl-2-O-benzyl-a-L-rhamnopyranosyloxy]-}

4H-chromen-4-one (10). To a stirred suspension containing $0.28 \mathrm{~g}(0.51 \mathrm{mmol})$ of 4, $0.24 \mathrm{~g}(1.02 \mathrm{mmol})$ of freshly prepared $\mathrm{Ag}_{2} \mathrm{O}$ and $4 \AA$ molecular sieves in $10 \mathrm{~mL}$ of $\mathrm{CH}_{2} \mathrm{Cl}_{2}$ was added $0.41 \mathrm{~g}(1.02 \mathrm{mmol})$ of 9 in 5 $\mathrm{mL}$ of $\mathrm{CH}_{2} \mathrm{Cl}_{2}$. The reaction mixture was stirred at room temperature for $4 \mathrm{~h}$, then diluted with $20 \mathrm{~mL}$ of $\mathrm{CH}_{2} \mathrm{Cl}_{2}$ and filtered through a Celite pad and washed with $\mathrm{CH}_{2} \mathrm{Cl}_{2}$. The filtrate was concentrated under diminished pressure and the residue was purified by flash chromatography on a silica gel column ( $25 \times 3$ $\mathrm{cm})$. Elution with 3:1 hexanes-ethyl acetate gave $\mathbf{1 0}$ as a colorless solid: yield $0.27 \mathrm{~g}(60 \%)$; silica gel TLC $R_{f} 0.37\left(2: 1\right.$ hexanes-ethyl acetate); $[\alpha]_{\mathrm{D}}{ }^{23}-84.3\left(c 0.06, \mathrm{CHCl}_{3}\right) ;{ }^{1} \mathrm{H} \mathrm{NMR}\left(\mathrm{CDCl}_{3}\right) \delta 0.87(\mathrm{~d}, 3 \mathrm{H}, J=6.0$ $\mathrm{Hz}), 1.95(\mathrm{~s}, 3 \mathrm{H}), 1.99(\mathrm{~s}, 3 \mathrm{H}), 3.39-3.48(\mathrm{~m}, 1 \mathrm{H}), 4.42(\mathrm{~m}, 1 \mathrm{H}), 4.68(\mathrm{~d}, 1 \mathrm{H}, J=12.3 \mathrm{~Hz}), 4.80(\mathrm{~d}, 1 \mathrm{H}, J=$ $12.3 \mathrm{~Hz}) ; 5.09(\mathrm{~s}, 2 \mathrm{H}), 5.14(\mathrm{~s}, 2 \mathrm{H}),[\mathrm{H}-2$ and H-3 protons underneath benzylic methylenes], $5.26(\mathrm{~s}, 2 \mathrm{H})$, 
$5.30(\mathrm{~s}, 2 \mathrm{H}), 5.74(\mathrm{~s}, 1 \mathrm{H}), 6.49(\mathrm{~s}, 1 \mathrm{H}), 6.58(\mathrm{~s}, 1 \mathrm{H}), 7.14(\mathrm{~d}, 2 \mathrm{H}, J=8.7 \mathrm{~Hz}), 7.25-7.47(\mathrm{~m}, 16 \mathrm{H}), 7.63(\mathrm{~d}$, $2 \mathrm{H}, J=7.8 \mathrm{~Hz})$ and $7.84(\mathrm{~d}, 2 \mathrm{H}, J=8.4 \mathrm{~Hz}) ;{ }^{13} \mathrm{C} \mathrm{NMR}\left(\mathrm{CDCl}_{3}\right) \delta 17.0,20.7,68.1,70.0,70.36,70.42,70.6$, $71.0,72.5,75.5,93.8,98.1,98.7,109.9,114.6,123.1,126.5,127.2,127.5,127.6,128.1,128.3,128.5,128.6$, $128.6,130.4,135.5,136.2,137.7,137.9,154.1,158.8,159.7,160.3,162.7,169.7,170.0$ and 173.0; mass spectrum $(\mathrm{FAB}), \mathrm{m} / \mathrm{z} 877.3221(\mathrm{M}+\mathrm{H})^{+}\left(\mathrm{C}_{53} \mathrm{H}_{49} \mathrm{O}_{12}\right.$ requires 877.3224).

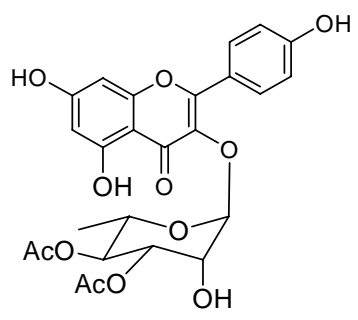

SL0101 (1). A suspension containing $0.26 \mathrm{~g}(0.29 \mathrm{mmol})$ of 10 and $0.10 \mathrm{~g}$ of $\mathrm{Pd}(\mathrm{OH})_{2} / \mathrm{C}$ in $10 \mathrm{~mL}$ of $1: 1$ THF-MeOH was purged with $\mathrm{H}_{2}$ and maintained under a $\mathrm{H}_{2}$ atmosphere for $1 \mathrm{~h}$. The reaction mixture was then diluted with ethyl acetate, filtered through a Celite pad and washed with ethyl acetate. The filtrate was concentrated under diminished pressure to give 1 as a tan solid: yield $0.14 \mathrm{~g}(94 \%)$; silica gel TLC $R_{f} 0.35$ (1:1:0.1 hexanes-ethyl acetate-methanol); $[\alpha]_{\mathrm{D}}{ }^{17}-106.5(c 0.65, \mathrm{MeOH}) ;{ }^{1} \mathrm{H}$ NMR (acetone- $\left.d_{6}\right) \delta 0.86(\mathrm{~d}$, $3 \mathrm{H}, J=6.3 \mathrm{~Hz}), 1.32($ br s, $1 \mathrm{H}), 2.01(\mathrm{~s}, 3 \mathrm{H}), 2.06(\mathrm{~s}, 3 \mathrm{H}), 3.54(\mathrm{~m}, 1 \mathrm{H}), 4.48(\mathrm{~d}, 1 \mathrm{H}, J=2.1 \mathrm{~Hz}), 5.12(\mathrm{~m}$, 1H), $5.23(\mathrm{dd}, 1 \mathrm{H}, J=9.9,2.7 \mathrm{~Hz}), 5.61(\mathrm{~d}, 1 \mathrm{H}, J=0.9 \mathrm{~Hz}), 6.32($ br s, 1H), 6.53 (br s, 1H), 7.09 (d, 2H, $J=$ 8.4 Hz), $7.90(\mathrm{~d}, 2 \mathrm{H}, J=8.1 \mathrm{~Hz}), 9.44(\mathrm{br} \mathrm{s}, 2 \mathrm{H})$ and $12.62(\mathrm{br} \mathrm{s}, 1 \mathrm{H}) ;{ }^{13} \mathrm{C}$ NMR (acetone- $\left.d_{6}\right) \delta 17.0,20.2$, $68.7,70.5,71.7,94.1,99.2,101.5,105.2,115.9,121.8,131.1,134.8,157.5,158.0,160.5,162.7,164.6$, 169.7, 170.1 and 178.5; mass spectrum (FAB), $\mathrm{m} / \mathrm{z} 517.1345(\mathrm{M}+\mathrm{H})^{+}\left(\mathrm{C}_{25} \mathrm{H}_{25} \mathrm{O}_{12}\right.$ requires 517.1346). 